TITLE:

\title{
Magnetic Light-Matter Interactions in a Photonic Crystal Nanocavity
}

\section{$\operatorname{AUTHOR}(\mathrm{S}):$}

Burresi, M.; Kampfrath, T.; van Oosten, D.; Prangsma, J. C.; Song, B. S.; Noda, S.; Kuipers, L.

\section{CITATION:}

Burresi, M. ... [et al]. Magnetic Light-Matter Interactions in a Photonic Crystal Nanocavity. Physical Review Letters 2010, 105(12): 123901.

\section{ISSUE DATE:}

2010

URL:

http://hdl.handle.net/2433/148382

RIGHT:

(C) 2010 The American Physical Society 


\title{
Magnetic Light-Matter Interactions in a Photonic Crystal Nanocavity
}

\author{
M. Burresi, ${ }^{1, *}$ T. Kampfrath, ${ }^{1}$ D. van Oosten, ${ }^{1}$ J. C. Prangsma, ${ }^{1}$ B. S. Song,${ }^{2,3}$ S. Noda,${ }^{2}$ and L. Kuipers ${ }^{1}$ \\ ${ }^{1}$ Center for Nanophotonics, FOM Institute for Atomic and Molecular Physics (AMOLF), \\ Science Park 104, 1098 XG Amsterdam, The Netherlands \\ ${ }^{2}$ Department of Electronic Science and Engineering, Kyoto University, Katsura, Nishikyo-ku, Kyoto 615-8510, Japan \\ ${ }^{3}$ School of Information and Communication Engineering, Sungkyunkwan University, Janan-Gu, \\ Suwon Gyeonggi-do 440-746, South Korea
}

(Received 30 March 2010; revised manuscript received 6 July 2010; published 17 September 2010)

\begin{abstract}
We study the magnetic coupling between a metal-coated near-field probe and a photonic crystal nanocavity. The resonance of the nanocavity shifts to shorter wavelengths when the ringlike apex of the probe is above an antinode of the magnetic field of the cavity. We show that this can be attributed to a magnetic light-matter interaction and is in fact a manifestation of Lenz's law at optical frequencies. We use these measurements to determine the magnetic polarizability of the apex of the probe and find good agreement with theory. We discuss how this method could be applied to study the electric and magnetic polarizibilities of nano-objects.
\end{abstract}

DOI: 10.1103/PhysRevLett.105.123901

Photonic crystals are materials which provide a high degree of control over the propagation of light at the nanoscale [1]. The most striking examples are photonic crystal nanocavities that can trap light in volumes comparable to the cubed wavelength for many optical cycles [2]. Such nanocavities currently have a broader linewidth compared to their macroscopic counterparts, that have been used, e.g., to trap single atoms [3] and to measure the size of nanoparticles [4]. However, as the mode volume of photonic crystal nanocavities is typically up to 5 orders of magnitude smaller than that of a microscale high-finesse cavity, one can still obtain a much higher sensitivity when studying the interaction of light with nano-objects.

Up to now, such investigations have focused on the coupling between matter and the electric component of light [4-6], as the material response to magnetic fields is usually negligible at frequencies above a few terahertz $[7,8]$. Recently, optical metamaterials have been developed whose subwavelength geometry induces a magnetic response at optical frequencies [9-13]. Given the resulting interest in the magnetic properties of materials at optical frequencies, a means to investigate magnetic light-matter interactions is desirable. Photonic crystal nanocavities are perfectly suited for such studies. Firstly, they are highly sensitive to external perturbations. Secondly, since the antinodes of the magnetic field inside the nanoresonator occur at the nodes of the electric field, the magnetic and electric response could be separately determined by measuring the cavity response as a function of the position of the nano-object. This technique could make it possible to measure the magnetic polarizibilities (i.e., the optical magnetic "size") of any nano-objects, not only metamaterials like split-ring resonators, but even smaller objects like quantum rings, carbon nanotubes, or atoms, whose magnetic properties at optical frequencies are unexplored.
PACS numbers: 42.70.Qs, 42.25.Bs, 42.82.-m, 78.67.Pt

In this work, we study how the resonance frequency of a photonic crystal nanocavity shifts when we bring a nearfield aperture probe in close proximity to the surface of the cavity. When the metallic ringlike aperture of the probe is above an antinode of the magnetic field, we observe that the cavity resonance shifts to shorter wavelengths. From this blueshift, we determine the magnetic polarizability of the near-field aperture and find excellent agreement with theory. Based on this result, we discuss the feasibility of this method to measure the magnetic polarizability of even smaller nano-objects.

The essential parts of our experiment are a nanocavity and a nanoprobe, as shown in the schematic of Fig. 1(a). The nanocavity consists of three missing holes in a twodimensional photonic crystal, which is a periodically per-
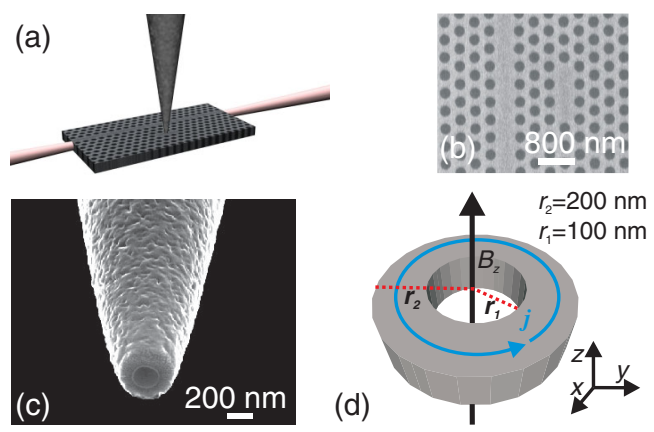

FIG. 1 (color). (a) Schematic of the experiment. Light is loaded into a photonic crystal nanocavity via a side-coupled photonic crystal waveguide. A metal-coated nanoprobe is used to perturb the cavity. (b) Scanning electron micrograph of the photonic crystal nanocavity and access waveguide. (c) Scanning electron micrograph of the aperture probe, which consists of a silica core coated by aluminum. (d) Schematic of the ring that models the apex of the probe. Here, $r_{1}=100 \mathrm{~nm}$ and $r_{2}=200 \mathrm{~nm}$ are the inner and outer ring radius, respectively, and $\boldsymbol{j}$ is the magnetically induced current density. 
forated, 250-nm-thick freestanding silicon membrane. The cavity is side coupled to a photonic crystal waveguide, which is a missing row of holes separated from the cavity by three rows of holes [Fig. 1(b)]. The resonance of the unperturbed cavity lies at a vacuum wavelength of $\lambda_{r_{0}}=$ $1534.6 \mathrm{~nm}$ and has a quality factor of $Q_{0}=6500$. We excite the cavity by coupling light of wavelength $\lambda$ from a continuous-wave diode laser into the access waveguide. A metal-coated near-field probe is placed in the evanescent field at a constant height of $20 \mathrm{~nm}$ above the sample surface [Fig. 1(a)]. The probe consists of a tapered single-mode optical fibre coated by a 100-nm-thick aluminum layer and treated with focused-ion-beam milling to obtain a dielectric aperture with a diameter of $200 \mathrm{~nm}$ [Fig. 1(c)]. In order to characterize the interaction between probe and cavity, we detect the transmittance of the access waveguide. By raster-scanning the probe over the cavity surface, the transmittance $T(x, y, \lambda)$ of the structure as a function of in-plane probe position $(x, y)$ and wavelength $\lambda$ is obtained.

In the following, we will analyze the normalized transmittance $\bar{T}(x, y, \lambda)=T(x, y, \lambda) / T_{0}(\lambda)$, where $T_{0}$ is the transmittance acquired when the perturbing probe is far away from the cavity. Note that $\bar{T}$ can exceed unity if the perturbed transmittance is larger than the unperturbed one. Figure 2(a) displays a map of $\bar{T}(x, y, \lambda)$ obtained at the resonance wavelength $\lambda_{r_{0}}$ of the unperturbed nanocavity. The blue regions indicate a decrease of transmittance whereas the yellow regions are associated with an increase. These variations can be understood by looking at Fig. 2(b) which depicts the normalized transmittance spectrum $\bar{T}\left(x_{0}, y_{0}, \lambda\right)$ for the probe position indicated by the white arrow in Fig. 2(a). We observe that for wavelengths around the unperturbed resonance at $\lambda_{r_{0}}$, the transmittance increases. Conversely, a dip of decreased transmittance is

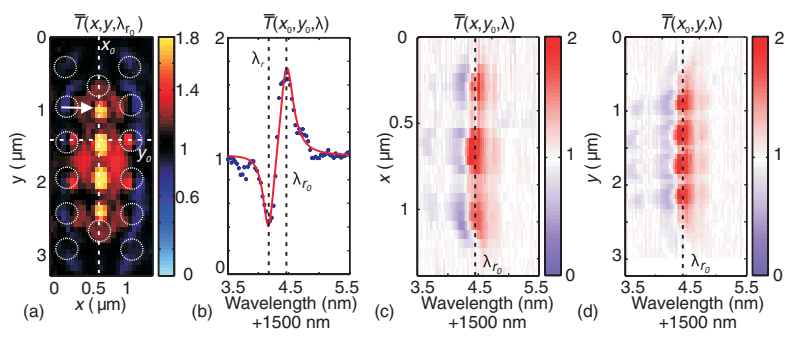

FIG. 2 (color). (a) In-plane map of the normalized waveguide transmittance $\bar{T}\left(x, y, \lambda_{r_{0}}\right)$ at the unperturbed cavity resonance. Black regions indicate unchanged transmission. The dashed circles indicate the position of the holes of the underlying photonic crystal structures. (b) Normalized transmittance spectrum $\bar{T}(x, y, \lambda)$ at probe position indicated by the white arrow in (a). The peak $(\bar{T}>1)$ indicates the resonance of the unperturbed cavity whereas the dip $(\bar{T}<1)$ arises from the new cavity resonance induced by the presence of the probe. A fit (red solid line) to the spectrum allows us to extract the probe-induced shift of the resonance. (c),(d) Probe-induced resonance shift for tip positions along the horizontal and vertical dashed lines in (a), respectively. A blueshift as indicated by the transmittance dip is evident for all probe positions. found approximately $0.4 \mathrm{~nm}$ below $\lambda_{r_{0}}$. This observation is a clear hallmark of a probe-induced shift $\lambda_{r_{0}} \rightarrow \lambda_{r}(x, y)$ of the cavity resonance: as the resonance wavelength shifts away from the laser wavelength, there is less coupling from the waveguide to the cavity. Less coupling, in turn, reduces the overall losses that the light experiences in the waveguide-cavity system and, thus, increases the transmittance at $\lambda_{r_{0}}$. On the other hand, at the new resonance wavelength $\lambda_{r}$, the waveguide-cavity coupling has increased, which implies larger losses and a reduced transmittance [6]. In other words, the peak and the dip of $\bar{T}$ in Fig. 2(b) roughly indicate the resonance of the unperturbed and perturbed cavity, respectively. Thus, a blueshift of the cavity resonance has been induced by the probe.

Qualitatively similar behavior is found at all probe positions. For example, Figs. 2(c) and 2(d) show $\bar{T}$ for probe positions along the horizontal and vertical dashed lines in Fig. 2(a), respectively. A decrease in transmittance (violet regions), indicating the induced resonance at $\lambda r$, can be only seen on the "blue side" of $\lambda_{r_{0}}$ (dashed lines), for all probe locations. Since no decrease of transmittance is discernible at wavelengths above $\lambda_{r_{0}}$ we can assert that a redshift is not observed.

The observed blueshift indicates a magnetic interaction between the light in the cavity and our probe. This can be understood by considering the probe as a metallic ring [Fig. 1(d)], whose height is given by the 100-nm-long evanescent tail of the cavity light field [14]. According to Faraday's law, the magnetic-field component $B_{z}$ induces a circular current in the ringlike probe apex. As described by Lenz's law, this current generates a magnetic field that opposes and, thus, reduces the driving field. As a consequence, the effective cavity volume shrinks, leading to a resonance shift towards higher frequencies and shorter wavelengths. In other words, the observed blueshift of the cavity resonance can be understood as a direct manifestation of Lenz's law at the nanoscale. As shown below, this interpretation is corroborated by the magnitude and by the dependence of the induced resonance shift $\Delta \lambda_{r}=$ $\lambda_{r}-\lambda_{r_{0}}$ on the probe position.

In order to obtain more quantitative information about $\Delta \lambda_{r}$ and a possible change in the quality factor, we apply coupled-mode theory to our measured transmittance data $\bar{T}$. When the waveguide and the cavity are treated as coupled elements, the waveguide transmittance is given by [15]

$$
T(x, y, \lambda)=1-\frac{1 / Q_{v}^{2}-1 / Q^{2}}{4\left(\lambda_{r} / \lambda-1\right)^{2}+1 / Q^{2}} .
$$

The total inverse quality factor is $1 / Q=1 / Q_{\text {in }}+1 / Q_{v}$, where $1 / Q_{\text {in }}$ and $1 / Q_{v}$ quantify the cavity losses to the inplane waveguide and the losses to free space in the vertical direction, respectively. Applying these relations to both the perturbed and the unperturbed cavity yields an expression for $T(x, y, \lambda) / T_{0}(\lambda)$ which is fitted to the measured normalized transmission spectrum $\bar{T}(x, y, \lambda)$ for each probe 
position $(x, y)$. The position-dependent fit parameters are $\lambda_{r}, Q_{\text {in }}$, and $Q_{v}$ whereas the values of $\lambda_{r_{0}}, Q_{\text {in } 0}$, and $Q_{v 0}$ are the same for all positions and wavelengths. The resulting fits are in excellent agreement with the measured data as demonstrated by the red line in Fig. 2(b).

The extracted relative shift $\Delta \lambda_{r} / \lambda_{r_{0}}$ of the cavity resonance is displayed in Fig. 3(a) as a function of probe position. Note that this map is consistent with the raw data $\bar{T}\left(x, y, \lambda_{r 0}\right)$ both in terms of the spatial distribution [Fig. 2(a)] and in terms of the magnitude of the resonance shift: in agreement with Fig. 2(b), a blueshift of up to $0.46 \mathrm{~nm}$ is found at certain probe positions. It is highly instructive to compare the map of $\Delta \lambda_{r} / \lambda_{r_{0}}$ of Fig. 3(a) to Figs. 3(b) and 3(c) which depict the modulus $\left|B_{z 0}\right|$ of the out-of-plane magnetic component and the modulus $\left|\boldsymbol{E}_{0}\right|$ of the total electric component of the unperturbed cavity field, respectively, as calculated by a three-dimensional finitedifference time-domain (FDTD) approach. Note that the spatial symmetry of the $\Delta \lambda_{r} / \lambda_{r_{0}}$ image closely and exclusively resembles that of $\left|B_{z 0}\left(x, y, \lambda_{r_{0}}\right)\right|$. We conclude that at probe positions with maximum magnetic field, a blue shift of the cavity resonance occurs. Moreover, since no features of the symmetry of $\left|\boldsymbol{E}_{0}\left(x, y, \lambda_{r_{0}}\right)\right|$ are discernible, the cavity-probe interaction appears to be dominated by the magnetic rather than the electric component of the cavity light field.

Our findings are in sharp contrast to previous works which all reported a probe-induced redshift of the cavity resonance at the antinodes of the electric field $[5,6,16]$. Note that in all these experiments much smaller tips with linear dimensions below the wavelength and the skin depth of the light were used. In this case [8], the perturbing tip can be described by an electric dipole. Far away from optical resonances of the tip, this dipole oscillates in phase with the exciting cavity field, which implies an increase of
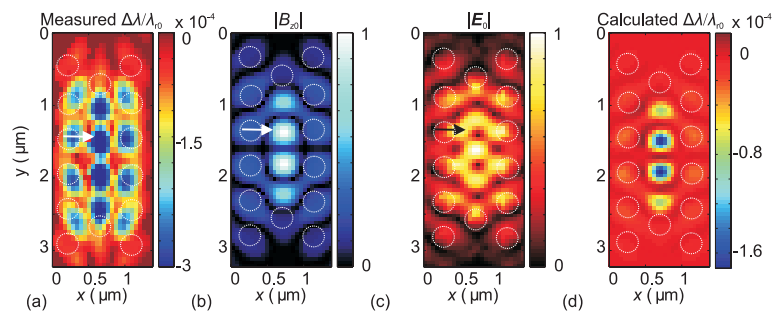

FIG. 3 (color). (a) In-plane map of the measured relative shift of the resonance wavelength of the cavity. (b) Calculated modulus $\left|B_{z 0}\right|$ of the out-of-plane magnetic component of the light field in the unperturbed cavity at $\lambda=\lambda_{r_{0}}$ as calculated by an FDTD approach. (c) Same as (b) for the modulus $\left|\boldsymbol{E}_{0}\right|$ of the total electric field. The arrows in (a), (b), and (c) indicate the measured resonance shift and calculated field that we use for estimating the magnetic polarizability of the probe. (d) Calculated relative shift in resonance wavelength based on Eq. (3), the FDTD-calculated and convoluted fields, and the calculated polarizabilities of the ring (see text). In all images, the dashed circles indicate the position of the holes of the underlying photonic crystal structure. the optical energy inside the cavity, $\Delta U>0$, and thus a redshift of the cavity resonance. As our tip is larger than the skin depth of the light and the metal coating has a tubelike shape, the induction of a pronounced magnetic dipole can be expected [8].

To put this discussion on a quantitative basis, we use perturbation theory [17] to calculate the induced relative resonance shift of the cavity as

$$
\frac{\Delta \lambda_{r}}{\lambda_{r_{0}}}=\frac{\Delta U}{U_{0}} .
$$

Here, $U_{0}=\left(2 / \mu_{0}\right) \int d^{3} \boldsymbol{x}\left|\boldsymbol{B}_{0}(\boldsymbol{x})\right|^{2}$ is the energy of the unperturbed cavity field, and $\Delta U=\left(\lambda_{r_{0}} / 2 \pi i c\right) \int d^{3} \boldsymbol{x} \boldsymbol{j}^{*} \cdot \boldsymbol{E}_{0}$ is the change in energy which arises from the current density $\boldsymbol{j}$ induced in the probe. In the dipole approximation, $\Delta U$ equals $\boldsymbol{E}_{0}^{*} \cdot \boldsymbol{p}+\boldsymbol{B}_{0}^{*} \cdot \boldsymbol{m}$, where the induced electric and magnetic dipole moments $\boldsymbol{p}=\alpha^{\mathrm{ee}} \boldsymbol{E}_{0}$ and $\boldsymbol{m}=$ $\alpha^{\mathrm{mm}} \boldsymbol{B}_{0}$ are connected to the fields through the electric and magnetic polarizability $\alpha^{\mathrm{ee}}$ and $\alpha^{\mathrm{mm}}$, respectively. Because of the cylindrical symmetry of the probe, there is no cross coupling between electric and magnetic quantities (in contrast to the probe employed in [14]). Moreover, the polarizability tensors are diagonal with $\alpha_{x x}^{\mathrm{ee}}=\alpha_{y y}^{\mathrm{ee}}$ and $\alpha_{x x}^{\mathrm{mm}}=\alpha_{y y}^{\mathrm{mm}}=0$. Since $\left|E_{z}\right| \ll\left|E_{x}\right|,\left|E_{y}\right|$, Eq. (2) turns into

$$
\frac{\Delta \lambda_{r}}{\lambda_{r_{0}}} \approx \alpha_{x x}^{\mathrm{ee}} \frac{\left|E_{0 x}^{2}\right|+\left|E_{0 y}^{2}\right|}{U_{0}}+\alpha_{z z}^{\mathrm{mm}} \frac{\left|B_{0 z}^{2}\right|}{U_{0}} .
$$

Equation (3) implies a negative $\alpha_{z z}^{\mathrm{mm}}$ since the observed maximum blueshift [Fig. 3(a)] occurs at locations where the magnetic field is maximum [Fig. 3(c)]. In order to extract values of $\alpha_{z z}^{\mathrm{mm}}$ from our experimental data, we use the measured $\Delta \lambda_{r} / \lambda_{r_{0}}$ [Fig. 3(a)] and the calculated fields of the unperturbed cavity [Figs. 3(b) and 3(c)] as input for Eq. (3). The value of $\alpha_{z z}^{\mathrm{mm}}$ is inferred at a probe position where $\left|B_{0 z}\right|$ is maximum and $\left|\boldsymbol{E}_{0}\right|$ vanishes [arrow in Figs. 3(a)-3(c)]. We obtain a magnetic polarizability $\alpha_{z z}^{\mathrm{mm}}=-12 \times 10^{-21} \mathrm{~m}^{3} / \mu_{0}$. This value agrees well with a theoretical estimate based on a quasistatic treatment [9], which yields $\alpha_{z z}^{\mathrm{mm}}=-7 \times 10^{-21} \mathrm{~m}^{3} / \mu_{0}$. Note that this estimate is independent of frequency and does not involve any resonance.

A similar quasistatic estimate of the electric polarizability models the ringlike tip apex as a perfectly conducting spheroid, a methodology often employed for split-ring resonators larger than the skin depth of light [18]. As the wavelengths considered here are far to the red of the plasmon resonance of the tip, the electric polarizability is positive and amounts to $\alpha_{x x}^{\mathrm{ee}}=3 \times 10^{-21} \mathrm{~m}^{3} \cdot \varepsilon_{0}$ [7]. This value is indeed smaller than the magnitude of the magnetic polarizability but still large enough to generate a detectable redshift at the maxima of the electric field. However, such a redshift is not observed [Fig. 3(a)]. We can understand the dominance of magnetic coupling between cavity and tip by taking the finite size of the probe into account. Note that 
the whole apex has a size about half the wavelength of the light inside the cavity. As a consequence, the fields spatially vary over the volume of the probe such that the effect of both the magnetic and electric interaction will be smeared out. We expect this convolution to be much more severe for the electric interaction since the relevant size is the whole area of the whole apex, whereas for the magnetic effect it is merely the area of the aperture. To model this finite-size effect, we convolute the FDTDcalculated electric and magnetic field with a disc of radius $r_{2}=200 \mathrm{~nm}$ and $r_{1}=100 \mathrm{~nm}$, respectively, prior to using Eq. (3). This procedure yields the shift of the cavity resonance as a function of probe position as displayed in Fig. 3(d). This map is in good agreement with the experimental observations in terms of number of antinodes, in terms of magnitude and in terms of the absence of a redshift.

As seen above, the resonance shift allows us to infer the magnetic polarizability of the aperture probe. In order to apply our scheme to other nano-objects, we have to discuss the sensitivity of this method. A polarizability is easily detected if the relative shift $\Delta \lambda / \lambda_{0}$ of the cavity resonance is larger than the relative cavity linewidth $1 / Q_{0}$. The cavity used in this work exhibits $1 / Q_{0}=1.5 \times 10^{-4}$, and the observed relative shift is $\Delta \lambda / \lambda_{0}=3 \times 10^{-4}$. Therefore, we can comfortably measure the magnetic polarizability of our near-field probe with this configuration. The sensitivity of the system can be increased by choosing a cavity with a higher quality factor. As cavities with a quality factor $Q>$ $10^{6}$ have recently become available [19], it should be possible to detect a relative shift of $\Delta \lambda / \lambda_{0}=1 / Q \approx 3 \times$ $10^{-7}$ and, therefore, to measure magnetic polarizabilities 3 orders of magnitude smaller than that of our probe, i.e., as small as $\left|\alpha^{\mathrm{mm}}\right|=6 \times 10^{-24} \mathrm{~m}^{3} / \mu_{0}$. For comparison, the near-resonant magnetic polarizability of a two-level system, such as an alkali atom, is typically a few times $10^{-23} \mathrm{~m}^{3} / \mu_{0}$ [20] and that of a typical split-ring resonator has recently been shown to be around $2 \times 10^{-20} \mathrm{~m}^{3} / \mu_{0}$ [21]. Thus, the method demonstrated here shows great promise to measure the magnetic and electric polarizabilities of many types of small objects ranging from single atoms, all the way up to the nanoscale.

In conclusion, we have shown that magnetic control of the resonance of a photonic crystal nanocavity can be achieved by means of a nanoscale metallic ring: when the ring is above an antinode of the magnetic field of light inside the cavity, the resonance of the nanocavity shifts to shorter wavelengths. This is a manifestation of Lenz's law at the nanoscale and at optical frequencies. Surprisingly, we find that the magnetic coupling between the cavity and the nanoring can increase the quality factor of the system by up to $50 \%$. This effect will be discussed in a forthcoming publication. Besides raising fundamental questions about the magnetic interaction between light and matter and stimulating further research on the electromagnetic properties of such a widely used near-field probe, our results demonstrate that photonic crystal nanocavities can be used to investigate the magnetic properties of specific nano-objects at optical frequencies. We believe that the development of magnetic metamaterials can greatly benefit from this characterization technique.

We wish to thank R. Esteban and J. Aizpurua for providing useful calculations and H. Schoenmaker for technical support. This work is part of the research program of the "Stichting voor Fundamenteel Onderzoek der Materie (FOM)", which is financially supported by the "Nederlandse organisatie voor Wetenschappelijk Onderzoek (NWO)". Support by the NWO (VICI grant) is gratefully acknowledged. This work is also supported by NanoNed, a nanotechnology program of the Dutch Ministry of Economic affairs.

Note added in proof-During finalization of the manuscript, the work of Vignolini et al. [22] came to our attention.

*burresi@amolf.nl

[1] J. D. Joannopoulos, S. G. Johnson, J. N. Winn, R. D. Meade, Photonic Crystals: Molding the Flow of Light (Princeton University Press, Princeton, 2008), 2nd ed..

[2] Y. Akahane, T. Asano, B. S. Song, and S. Noda, Nature (London) 425, 944 (2003).

[3] T. Wilk, S. C. Webster, A. Kuhn, and G. Rempe, Science 317, 488 (2007).

[4] J. Zhu, et al., Nat. Photon. 4, 46 (2010).

[5] A. F. Koenderink, M. Kafesaki, B. C. Buchler, and V. Sandoghdar, Phys. Rev. Lett. 95, 153904 (2005).

[6] L. Lalouat, et al., Phys. Rev. B 76, 041102 (2007).

[7] L.D. Landau, E.M. Lifshitz. Electrodynamics of Continuous Media (Wiley, New York, 1984).

[8] R. Merlin, Proc. Natl. Acad. Sci. U.S.A. 106, 1693 (2009).

[9] J. B. Pendry, A. J. Holden, D. J. Robbins, and W. J. Stewart, IEEE Trans. Microwave Theory Tech. 47, 2075 (1999).

[10] J. B. Pendry, D. Schurig, and D. R. Smith, Science 312, 1780 (2006).

[11] G. Dolling et al., Science 312, 892 (2006).

[12] J. Valentine et al., Nature (London) 455, 376 (2008).

[13] N. Liu, H. Liu, S. Zhu, and H. Giessen, Nat. Photon. 3, 157 (2009).

[14] M. Burresi et al., Science 326, 550 (2009).

[15] C. Manolatou et al., IEEE J. Quantum Electron. 35, 1322 (1999).

[16] F. Intonti et al., Phys. Rev. B 78, 041401 (2008).

[17] S. G. Johnson et al., Appl. Phys. B 81, 283 (2005).

[18] R. Marques, F. Medina, and R. Rafii-El-Idrissi, Phys. Rev. B 65, 144440 (2002).

[19] T. Asano, B. S. Song, Y. Akahane, and S. Noda, IEEE J. Sel. Top. Quantum Electron. 12, 1123 (2006).

[20] R. Grimm, M. Weidemuller, and Y.B. Ovchinnikov. Advances in Atomic Molecular and Optical Physics (Academic Press, San Diego, 2000), Vol. 42.

[21] I. Sersic, M. Frimmer, E. Verhagen, and A. F. Koenderink, Phys. Rev. Lett. 103, 213902 (2009).

[22] S. Vignolini et al., following Letter Phys. Rev. Lett. 105, 123902 (2010) 